\title{
Les microalgues
}

\section{Usines cellulaires \\ productrices de molécules commerciales recombinantes}

> Le champ d'application des microalgues a longJean-Paul Cadoret, Muriel Bardor, Patrice Lerouge, temps été contenu dans les domaines de l'aquaMorgan Cabigliera, Vitalia Henriquez, Aude Carlier culture, de l'alimention pour animaux d'élevage, voire de complément pour l'alimentation humaine. Les récents progrès accomplis en recherche fondamentale et appliquée ont fait entrer les microalgues dans l'ère de la génomique. Au-delà des compétences acquises dans les systèmes de culture et la création de photobioréacteurs aux géométries originales, la transformation génétique est une réalité pour plusieurs dizaines de microalgues issues de groupes phylogénétiques différents. Un panel de vecteurs d'expression génétique est disponible et de nombreux autres sont en cours d'élaboration. Même si des savoir-faire solides restent encore à établir pour parvenir à l'expression de quantités raisonnables de molécules recombinantes, les avancées actuelles justifient l'intérêt de sociétés de biotechnologies et la création de start-up mettant en œuvre cette stratégie «usine cellulaire» chez les microalgues. <

L'extraction de substances naturelles constitue la source principale d'approvisionnement pour nombre de molécules pharmaceutiques. Dans la mesure où il est possible d'identifier les gènes codant pour une molécule, ceux-ci peuvent être introduits dans des cellules en culture qui deviennent alors autant d'usines cellulaires fabriquant le produit souhaité. Dans la dynamique créée par l'arrivée de nouveaux systèmes d'expression végétaux, les microalgues, organismes unicellulaires aquatiques, apportent des compléments stratégiques et devraient constituer à l'avenir un système performant de production de molécules recombinantes commerciales.

\section{Les différents systèmes d'expression}

À ce jour, plusieurs systèmes d'expression de protéines recombinantes ont été développés, offrant chacun des avantages et des inconvénients. Pour l'expression de molécules simples, les bactéries suffisent; c'est le cas, par exemple, de l'hormone de croissance, de l'insuline,

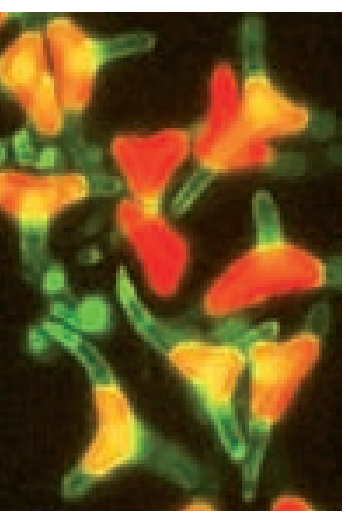

J.P. Cadoret, A. Carlier :

Laboratoire PBA, IFREMER, Centre de Nantes,

rue de l'lle d'Yeu, BP 21105, 44311 Nantes Cedex 03, France. jean.paul.cadoret@ifremer.fr M. Bardor, P. Lerouge : Université de Rouen, CNRS UMR 6037, IFRMP 23, Faculté des Sciences,

des interférons $\alpha$ et $\beta$, de l'interleukine-1 et du TNF (tumor necrosis factor) [1]. Cependant, ces organismes ne peuvent pas assembler des molécules complexes ni assurer leur maturation via des modifications post-traductionnelles comme les glycosyla76821 Mont-Saint-Aignan Cedex, France.

M. Cabigliera: Algenics, 5 rue d'Auvours, 44000 Nantes, France. V. Henriquez : Laboratorio de Genética e Inmunología Molecular, Instituto de Biologia, Pontificia Universidad Catolica de Valparaiso P0 Box 4059, Valparaiso, Chili. tions, l'isomérisation cis/trans des prolines, les modifications de lipidation ou de phosphorylation. La présence de corps d'inclusion qui rendent difficile la purification, et la production d'endotoxines complètent cette liste de défauts. En conséquence, la production de protéines recombinantes complexes requiert l'usage de systèmes d'expression eucaryotes [2].

\section{L'obstacle de la $\mathrm{N}$-glycosylation}

Parmi les modifications post-traductionnelles, la N-glycosylation revêt une importance particulière car elle a un impact majeur sur la conformation, et par conséquent sur l'activité, des protéines thérapeutiques produites par transgenèse. Ce processus débute par le transfert d'un précurseur oligosaccharidique sur un résidu asparagine de la protéine néo-synthétisée, suivi d'une maturation 
dans le réticulum endoplasmique puis d'une étape de modification des $\mathrm{N}$-glycanes par transfert de nouveaux résidus dans l'appareil de Golgi. Ces modifications golgiennes diffèrent entre cellules eucaryotes et il en résulte des différences structurales entre $\mathrm{N}$-glycanes associés aux protéines matures. En conséquence, si tout système eucaryote est apte à introduire des $\mathrm{N}$-glycanes indispensables à la mise en conformation des protéines, les différences de maturation de ceux-ci peuvent limiter ou interdire l'emploi en thérapie des molécules recombinantes, notamment en raison du risque de déclenchement d'une réponse immunitaire. Ainsi, les levures (Saccharomyces cerevisiae, Pichia pastoris, Kluyveromyces lactis et Hansenula polymorpha, [1]) introduisent sur leurs glycoprotéines des polymannoses de grande taille [3]. Le système d'expression en cellules d'insectes, via un Baculovirus, constitue un procédé attrayant de production de protéines par transgenèse. Les cultures croissent en suspension et la plupart des protéines surexprimées dans les cellules d'insectes restent solubles [1]. Cependant, la sialylation des glycoprotéines, étape ultime de la glycosylation, aboutit rarement. De même, la production dans des cellules animales ( $\mathrm{CHO}$, BHK et $\left.\mathrm{MML}^{1}\right)$ [1] se heurte également à des problèmes de sialylation terminale des $\mathrm{N}$-glycanes [4]. Quel que soit le système considéré, plusieurs stratégies ont été développées pour corriger les différences observées de glycosylation entre protéines naturelles et recombinantes, soit par l'inactivation de gènes codant pour des enzymes introduisant les résidus immunogènes, soit par complémentation par des gènes codant des enzymes permettant de reconstruire les séquences glucidiques absentes dans ces organismes [5]. Les résultats les plus spectaculaires ont été obtenus par Gerngross et ses collaborateurs, au sein de la société GlycoFi (États-Unis), qui ont complètement «humanisé » la N-glycosylation chez la levure par la suppression de 4 gènes et l'ajout de 14 autres [6].

\section{Du tabac à la mousse transgénique : avantages et inconvénients des cellules végétales}

Les cellules végétales peuvent, quant à elles, procéder à un repliement et à l'assemblage de protéines recombinantes complexes, ainsi qu'au transfert et à la maturation de $\mathrm{N}$-glycanes sur celles-ci. De plus, les organismes photosynthétiques offrent deux voies biologiques très différentes dans l'expression et la production de molécules recombinantes: une voie nucléaire et une voie chloroplastique (Tableau I). Les travaux pionniers de Hiatt avec la production d'anticorps fonctionnels exprimés dans du tabac transgénique, ont consacré le terme de Plantibodies (ou planticorps) [7]. De nombreuses autres molécules recombinantes actives, vaccins, anticorps monoclonaux, enzymes et hormones, ont été depuis produites avec succès [8]. Plusieurs plantes sont concernées, le tabac, le riz, le blé, la luzerne, le pois par exemple. Mais les cellules de plantes introduisent des épitopes glucidiques $\beta 1,2$-xylose et $\alpha 1,3$-fucose immunogènes chez l'humain [9] nécessitant des interventions génétiques [10]. Les premières commercialisations de molécules issues de plantes génétiquement modifiées ont concerné l'avidine, produite dans le maïs en 1997, la $\beta$-glucuronidase en 1998 ou plus récemment, la trypsine. Le risque de dissémination de gènes

${ }^{1}$ CHO : chinese hamster ovary; BHK : baby hamster kidney; MML : murine myeloma lines. dans le milieu naturel reste le principal écueil à l'essor de la production de molécules recombinantes chez les végétaux supérieurs, bien que les stratégies de transformation des chloroplastes (voir Encadré) tentent de répondre à cette question. À la grande variation des niveaux d'expression d'une plante à une autre s'ajoute l'instabilité de l'expression dans les feuilles, ce qui nécessite une extraction et une purification rapide avant d'éventuelles dégradations. De plus, la réaction du public vis-à-vis des plantes génétiquement modifiées demeure imprévisible. Pour pallier cet obstacle, la production de ces molécules recombinantes par des cellules végétales se fait en bioréacteurs confinés et sûrs. La détection des transgènes est rapide et l'expression des molécules recombinantes peut se faire dans le milieu de culture. Néanmoins, les rendements restent faibles et les effets mécaniques fragilisants.

\section{CHLOROPLASTES}

Les chloroplastes sont ces organites observés dans les cellules végétales et qui contiennent notamment la chlorophylle. C'est le lieu de la photosynthèse, responsable de la transformation de l'énergie lumineuse en ATP et substances nutritives par le biais d'une cascade d'oxydations. D'une taille de quelques microns, ils possèdent un $A D N$ circulaire leur permettant de se dupliquer de façon autonome et qui, de ce fait, font l'objet de recherche en génie génétique pour l'expression de molécules recombinantes.

Autre exemple de végétal, la mousse (Physcomytrella patens) transgénique. Des lignées transformées stables sont obtenues en 8 semaines et le biais de codon ${ }^{2}$ serait plus proche de l'animal que des plantes dressées $^{3}$. L'obstacle de l'immunogénicité induite par leur $\mathrm{N}$-glycosylation a été contourné par l'extinction des gènes codant pour les enzymes responsables de l'ajout des épitopes glucidiques immunogènes $(\beta 1,2$-xylose et $\alpha 1,3$-fucose). Ces travaux ont récemment abouti à l'expression d'érythropoïétine (EPO) humaine [11]. Autre exemple, la lentille d'eau Lemna (plante clonale utilisée par la société Biolex) possède un temps de génération court, un rendement élevé en protéines et un système d'excrétion permettant une production de protéines à faible coût. L'immunogénicité des $\mathrm{N}$-glycanes a également pu être abolie chez cette plante [12].

${ }^{2}$ Les triplets de nucléotides offrent 64 combinaisons pour 20 acides aminés, l'utilisation et le choix de ces combinaisons sont appelés biais de codon.

${ }^{3}$ Les plantes dressées sont caractérisées par une croissance verticale pour l'axe principal et par une croissance oblique (plagiotrope) pour les ramifications; on les distingue des plantes grimpantes et des plantes rampantes. 


\section{Les microalgues}

Les microalgues vivent de facto en suspension. Des centaines de milliers d'espèces de microalgues sont réparties sur la surface du globe en milieu marin, dulçaquicole 4 ou saumâtre (Figures 1-4). Elles ont colonisé les glaces polaires, les zones désertiques et les sources d'eau chaude. Elles

${ }^{4}$ Dulçaquicole qualifie un organisme qui vit dans les eaux douces. Saumâtre qualifie une eau constituée d'un mélange d'eau douce et d'eau de mer. Sa salinité peut être variable, mais est le plus souvent assez basse. (source: Ifremer).

\begin{tabular}{|c|c|c|}
\hline & Génome chloroplastique & Génome nucléaire \\
\hline Nombre de copies de transgène & $\begin{array}{l}\text { 10-100 copies de génome (chromosome circulaire } \\
\text { simple) par chloroplaste, aboutissant à plusieurs } \\
\text { copies de transgènes }\end{array}$ & $\begin{array}{l}\text { Le nombre de chromosomes est spécifique d'une } \\
\text { espèce, et chacune des deux copies des chromosomes } \\
\text { présents dans la cellule peut intégrer plusieurs copies } \\
\text { du transgène }\end{array}$ \\
\hline Niveau d'expression du gène & $\begin{array}{l}\text { La polyploïdie résulte en une abondance de trans- } \\
\text { crits transgéniques et en une grande accumulation } \\
\text { de protéines étrangères (jusqu'à } 47 \% \text { des protéines } \\
\text { solubles totales) }\end{array}$ & $\begin{array}{l}\text { La régulation des gènes détermine le taux de } \\
\text { transcription. L'accumulation de protéines étrangères } \\
\text { peut être une limitation }\end{array}$ \\
\hline $\begin{array}{l}\text { Arrangement et transcription } \\
\text { de gènes }\end{array}$ & $\begin{array}{l}\text { Les gènes peuvent être arrangés en opérons afin } \\
\text { d'introduire et d'exprimer de multiples transgènes } \\
\text { lors d'un seul événement de transformation }\end{array}$ & $\begin{array}{l}\text { Chaque transgène est inséré indépendamment dans } \\
\text { le chromosome }\end{array}$ \\
\hline Effet de positionnement & $\begin{array}{l}\text { Les insertions spécifiques de sites au travers de deux } \\
\text { événements de recombinaison homologue éliminent } \\
\text { les effets de positionnement de l'expression du } \\
\text { transgène }\end{array}$ & $\begin{array}{l}\text { Les insertions aléatoires résultent en des niveaux } \\
\text { d'expression transgéniques variables }\end{array}$ \\
\hline « Gene silencing 》 & Non documenté & $\begin{array}{l}\text { Le «gene silencing» conduit à une diminution ou } \\
\text { élimination de l'expression des transgènes. Des } \\
\text { problèmes de transcription et de post-transcription } \\
\text { de gènes inhibés sont rapportés }\end{array}$ \\
\hline Confinement de gènes & $\begin{array}{l}\text { Les gènes sont transmis par la voie maternelle } \\
\text { évitant une dissémination du gène par le pollen }\end{array}$ & $\begin{array}{l}\text { Les transgènes hérités peuvent être disséminés par } \\
\text { croisement entre les plantes cultivées et plantes } \\
\text { sauvages }\end{array}$ \\
\hline $\begin{array}{l}\text { Formation de ponts disulfures } \\
\text { et intégration }\end{array}$ & $\begin{array}{l}\text { Les chloroplastes forment des ponts disulfures et } \\
\text { expriment correctement des protéines d'origine } \\
\text { humaine, les rendant idéales pour le développement } \\
\text { de vaccins comestibles, la pharmaceutique et les } \\
\text { «planticorps» }\end{array}$ & $\begin{array}{l}\text { Pour la formation de ponts disulfures, les protéines } \\
\text { sont ciblées au niveau du réticulum endoplasmique }\end{array}$ \\
\hline $\begin{array}{l}\text { Protéines toxiques } \\
\text { et étrangères }\end{array}$ & $\begin{array}{l}\text { Les effets létaux des protéines toxiques peuvent } \\
\text { être minimisés grâce à la compartimentation du } \\
\text { chloroplaste }\end{array}$ & $\begin{array}{l}\text { L'accumulation de protéines toxiques à l'intérieure } \\
\text { du cytosol peut provoquer de sérieux effets } \\
\text { pléiotropiques }\end{array}$ \\
\hline Lignées transgéniques & Expression du gène uniforme & Expression du gène hautement variable \\
\hline Homogénéité de la ploïdie & $\begin{array}{l}\text { Les lignées transgéniques chloroplastiques sont } \\
\text { généralement homoplasiques (l'ensemble des copies } \\
\text { génomiques sont homogènes pour le transgène). } \\
\text { L’homoplasie est généralement complétée par une } \\
\text { sélection répétitive }\end{array}$ & $\begin{array}{l}\text { Les lignées transgéniques nucléaires sont } \\
\text { hétérozygotes ou homozygotes }\end{array}$ \\
\hline Glycosylation & Semble absente & $\begin{array}{l}\text { Les cellules eucaryotes végétales glycosylent mais selon } \\
\text { des schémas légèrement différents des mammifères }\end{array}$ \\
\hline
\end{tabular}

Tableau l. Comparaison des deux voies d'expression : chloroplastique et nucléaire chez les plantes. 


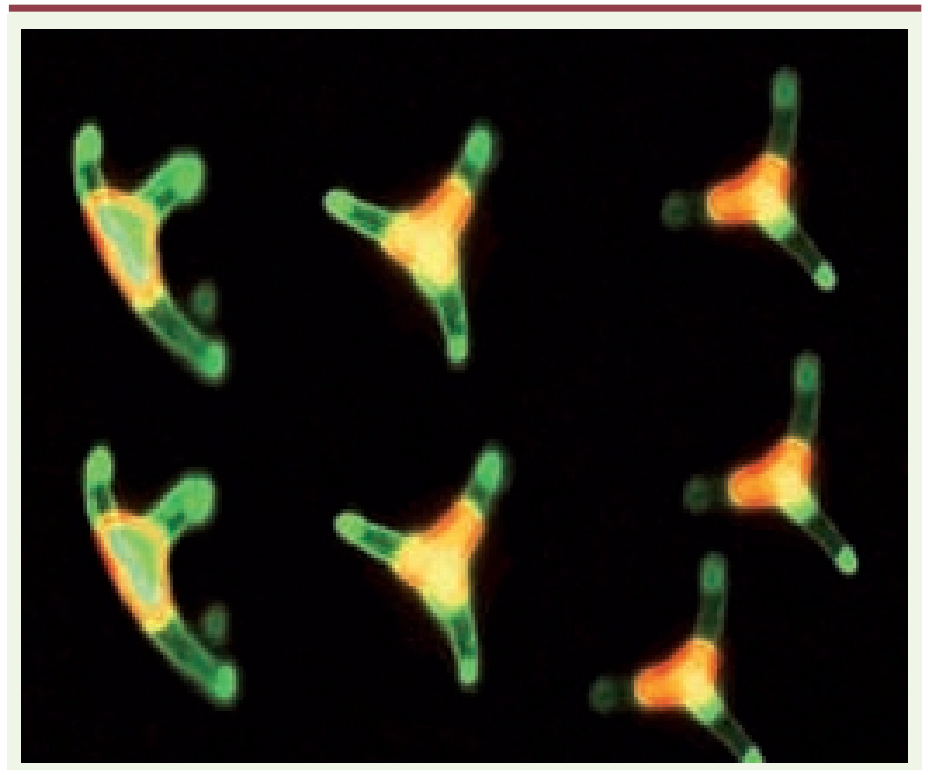

Figure 1.

vivent dans des marais salants, dans des milieux acides ou fortement alcalins. Elles jouent un rôle majeur dans le climat mondial comme machine à transformer le $\mathrm{CO}_{2}$ en matière organique. Leur culture en photobioréacteurs confinés est maîtrisée, réduisant les risques de dissémination et de pollution génique. Elles procurent d'excellents rendements en biomasse et restent compétitives en coûts de production. Elles n'exigent pour leur développement que de l'eau (douce ou salée), des éléments nutritifs, du carbone et de la lumière. Le taux de division cellulaire rapide (de l'ordre du jour) permet d'atteindre des densités cellulaires élevées et de répondre rapidement à toute stratégie de recherche et développement. L'activité d'une nouvelle construction génétique transférée dans ces organismes peut être recherchée et mesurée un mois après la mise en culture, et un volume conséquent (300 litres) peut être atteint deux mois plus tard, représentant une biomasse très importante ( 1 à 3 grammes sec par litre).

\section{Les microalgues, usines cellulaires}

La transformation génétique chez les microalgues a fait son apparition dans les années 1980. Les espèces phares sont alors Chlamydomonas reinhardtii, Dunaliella salina, Phaeodactylum tricornutum (voir Figures 1 à 4, p. 378 et 380) et Chorella vulgaris (Tableau II). Les perspectives et le potentiel des microalgues comme usines cellulaires ont été abordés et traités par différents auteurs, récemment et de façon plus ou moins

${ }^{5}$ Action complémentaire indispensable aux voies chimiques et physiques pour la dégradation ultime des hydrocarbures dans l'environnement. exhaustive [13-17]. L'algue verte unicellulaire Chlamydomonas reinhardtii s'est vite imposée comme système modèle [18]. Elle peut être cultivée de façon auto et hétérotrophe en utilisant de l'acétate comme source de carbone. Des séquences promotrices nucléaires et chloroplastiques ont été identifiées. Actuellement, C. reinhardtii n'est plus seulement un modèle d'étude fondamentale [18] mais est au cœur d'applications biotechnologiques avec la production de biohydrogène et la biorémédiation ${ }^{5}$ des sols pollués par des métaux lourds. Parmi les autres microalgues utilisées dans un cadre biotechnologique, citons l'expression transitoire de l'hormone de croissance humaine dans le milieu extracellulaire de Chlorella sp. Des soles alimentées avec Chlorella ellipsoidea, exprimant une hormone de croissance de sole, atteignent une taille de $25 \%$ supérieure à celle de poissons témoins après 10 jours de culture. Par ailleurs, des tests antimicrobiens in vitro ont montré la grande efficacité d'expression par les cellules de chlorella, du peptide neutrophile I (NP1 ), biologiquement actif. L'expression du gène VPI du virus de la fièvre aphteuse chez Chlamydomonas reste le seul exemple de production de protéine vaccinale (Tableau III). En terme de production de protéines thérapeutiques, le résultat le plus marquant est à ce jour la

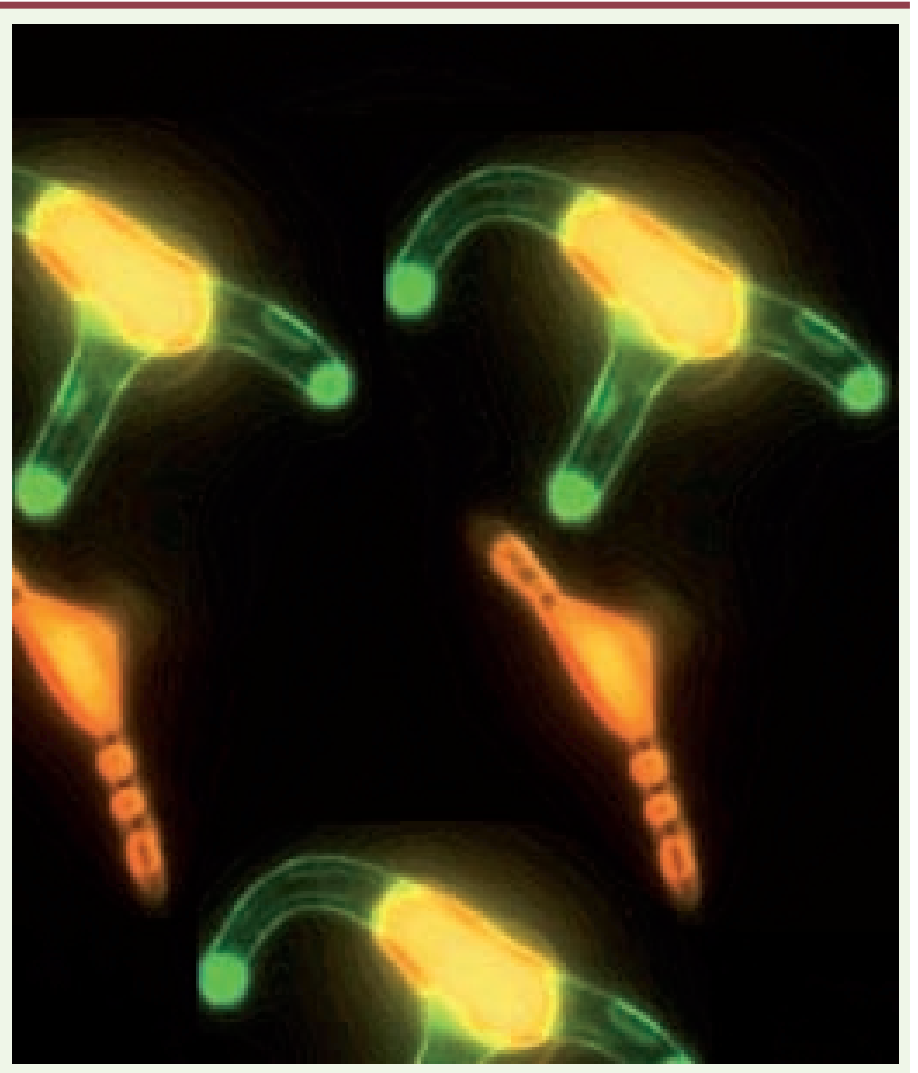

Figure 2. 
production de large single chain (Isc) d'anticorps dans la microalgue Chamydomonas reinhardtii par I 'équipe de S. Mayfield (Tableau III). Les processus de $\mathrm{N}$-glycosylation chez les microalgues n'ont pas à ce jour été explorés. Compte tenu de leur importance dans le cadre de la production de molécules recombinantes, nous avons entrepris une étude de ces processus chez plusieurs microalgues pressenties comme potentielles usines cellulaires. «L'ancestralité » de plusieurs groupes phylogénétiques de phytoplancton laisse espérer la conservation chez ces organismes des caractéristiques primitives des

\begin{tabular}{|c|c|c|c|}
\hline Microalgue & Méthode de transformation & Génome & Référence \\
\hline \multicolumn{4}{|l|}{ Microalgues vertes } \\
\hline \multirow[t]{7}{*}{ Chlamydomonas reinhardtii } & Bombardement de microparticules & Nucléaire & {$[20]$} \\
\hline & & Chloroplastique & [21] \\
\hline & & Mitochondrial & [22] \\
\hline & Électroporation & Nucléaire & [23] \\
\hline & Billes de verre & Nucléaire & {$[24]$} \\
\hline & Trichites de carbure de silicium & Nucléaire & {$[25]$} \\
\hline & Agrobacterium tumefaciens & Nucléaire & {$[26]$} \\
\hline Dunaliella salina & Électroporation & Nucléaire & {$[27]$} \\
\hline Dunaliella salina & Bombardement de microparticules & Nucléaire & {$[28]$} \\
\hline Chlorella ellipsoida & Polyéthylène glycol & Nucléaire & [29] \\
\hline Chlorella sorokiniana & Bombardement de microparticules & Nucléaire & {$[30]$} \\
\hline Chlorella vulgaris & Electroporation & Nucléaire & {$[31]$} \\
\hline Haematococcus pluvialis & Bombardement de Microparticules & Nucléaire & [32] \\
\hline Volvox carteri & Bombardement de microparticules & Nucléaire & [33] \\
\hline \multicolumn{4}{|l|}{ Diatomées } \\
\hline Cyclotella criptyca & Bombardement de microparticules & Nucléaire & {$[34]$} \\
\hline Navicula saprophila & Bombardement de microparticules & Nucléaire & [34] \\
\hline Phaeodactylum tricornutum & Bombardement de microparticules & Nucléaire & {$[35]$} \\
\hline Cylindrotheca fusiformes & Bombardement de microparticules & Nucléaire & {$[36]$} \\
\hline Thallassiosira pseudonana & Bombardement de microparticules & Nucléaire & [37] \\
\hline \multicolumn{4}{|l|}{ Dinoflagellés } \\
\hline Amphidinium spp. & Trichites de carbure de silicium & Nucléaire & [38] \\
\hline Symbiodinium microadriaticum & Trichites de carbure de silicium & Nucléaire & {$[38]$} \\
\hline \multicolumn{4}{|l|}{ Algues rouges } \\
\hline Cyanidoschyzon merolae & Electroporation & Nucléaire & [39] \\
\hline Porphyidium sp. & Bombardement de microparticules & Chloroplastique & {$[40]$} \\
\hline \multicolumn{4}{|l|}{ Euglenoids } \\
\hline Euglena gracilis & Bombardement de microparticules & Chloroplastique & {$[41]$} \\
\hline
\end{tabular}

Tableau II. Microalgues transformées et méthodes utilisées. 


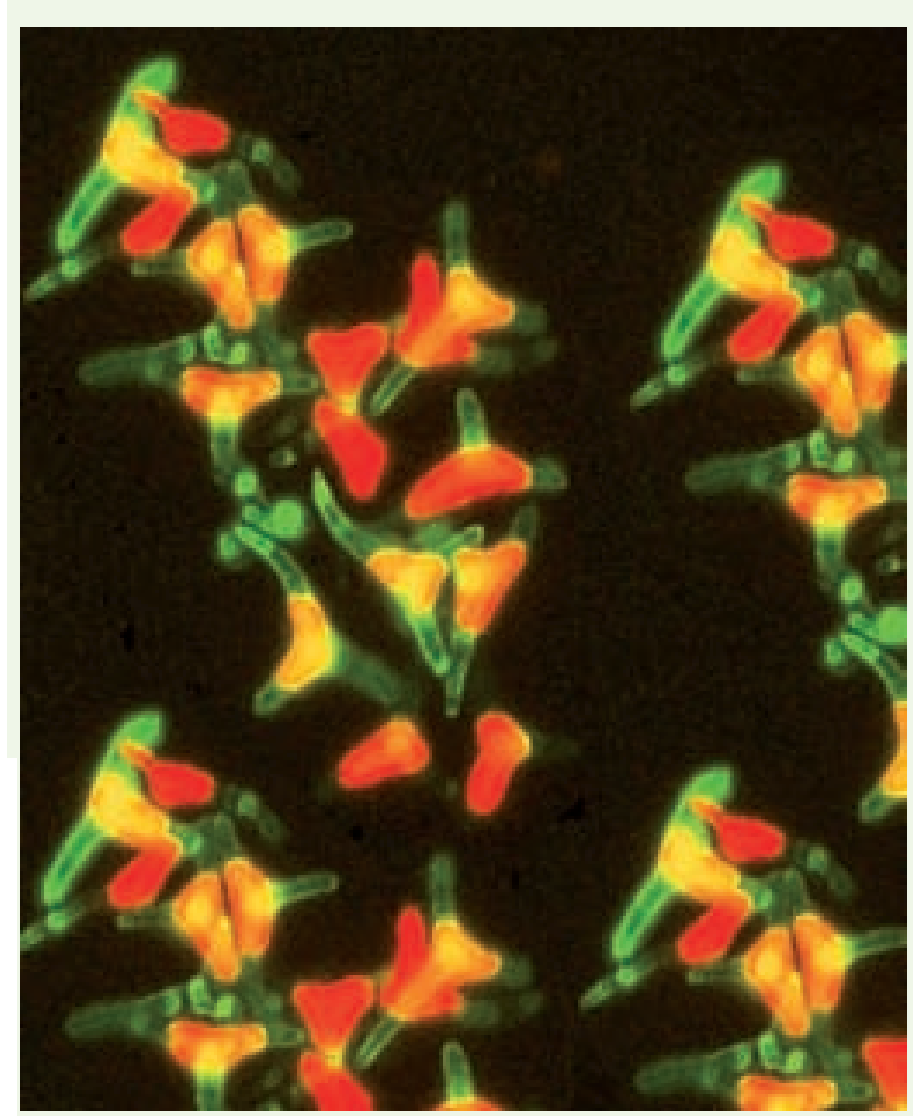

Figure 3.

processus de glycosylation, en amont du point de divergence entre les plantes supérieures et les animaux. On pourrait ainsi éluder l'obligatoire et difficile «adaptation » des processus de glycosylation.

La composition en nucléotides varie grandement entre genres, et dans une moindre mesure entre espèces voire dans un même génome. À titre d'exemple, pour le génome nucléaire des diatomées, le pourcentage est proche de l'équilibre : 49,63\% chez Phaeodactylum tricornutum, Thalassiosira sp., alors que des algues de la lignée verte telle Chlorella vulgaris comptent 63,92\% de GC, proche des $63 \%$ de Chlamydomonas reinhardtii. Les algues rouges, si l'on se réfère à Porphyridium cruentum $(59,4 \%)$ seront proches des algues vertes, les plantes terrestres étant en dessous de la barre moyenne puisque Arabidopsis thaliana présente un taux en GC de $44,60 \%$ et le tabac $43,57 \%$. Cet équilibre revêt une importance particulière dans le cas de l'expression de protéines hétérologues d'origine humaine, puisque Homo sapiens privilégie un usage des nucléotides GC à 52,4\%. II faut noter la relative homogénéité du taux de GC de tous les plastides quelles que soient les microalgues considérées avec pour Phaeodactylum sp.
$(33,69 \%)$, Thalassiosira sp. (30,17\%), Chlorella vulgaris $(34,63 \%)$, Chlamydomonas reinhardtii $(35,16 \%)$, Dunaliella sp (32,62\%), Tetraselmis sp. (39,18\%), Porphyridium cruentum $(36,51 \%)$.

\section{Les contraintes techniques}

À l'exception de méthodes anecdotiques comme la transformation par billes de verre de Chlamydomonas reinhardtii, la transformation par des trichites de carbure de silicium ( $\mathrm{SiC}$ ) ou la transformation par Agrobacterium chez Chlamydomonas reinhardtii, l'électroporation (consistant à soumettre les cellules à un champ électrique pour former des pores) est l'une des premières méthodes mise en œuvre avec succès pour la transformation nucléaire de quatre espèces de microalgues, Chlamydomonas reinhardtii, Chlorella ellipsoidea, Chlorella vulgaris et Dunaliella salina. Le bombardement par microparticules ou biolistique utilise des microparticules inertes d'or ou de tungstène recouvertes d'ADN et propulsées à grande vitesse par un gaz comprimé. C'est la méthode utilisée pour Chlamydomonas, Chlorella et Volvox. Elle s'est aussi montrée particulièrement efficace pour la transformation de diatomées (Tableau II).

Dans la liste des promoteurs utilisés chez les microalgues, on trouve le très classique promoteur du gène $35 \mathrm{~S}$ du virus de la mosaïque du chou-fleur, utilisé avec succès chez Chlorella ellipsoidea [19]. A mentionner, le promoteur de nitrate reductase endogène, actif chez Chlamydomonas

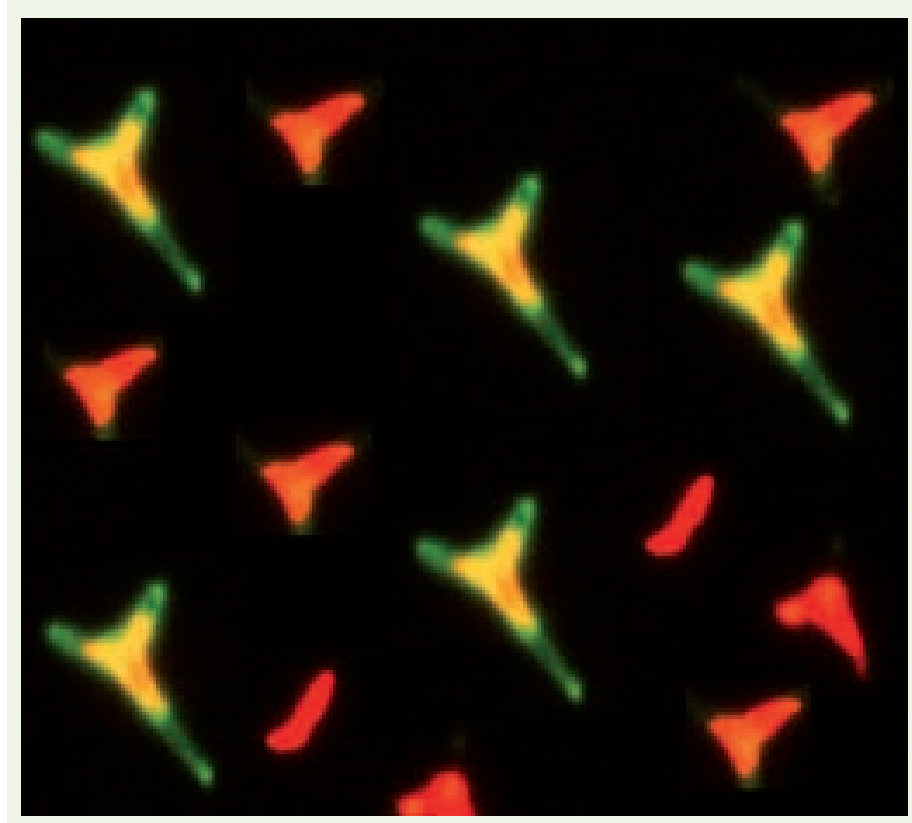

Figure 4. 


\begin{tabular}{lll}
\hline Protéine thérapeutique & Microalgues & Références \\
IgAl (virus de l'herpès) & Chlamydomonas & {$[15]$} \\
HSV Isc & reinhardtii & {$[42]$} \\
M-SAA & {$[43]$} \\
\hline Hormone de croissance & Chlorella ellipsoidea & {$[19]$} \\
\hline Antigène & \\
\hline & \\
\hline VPl fièvre aphteuse & $\begin{array}{l}\text { Chlamydomonas } \\
\text { reinhardtii }\end{array}$ & {$[44]$} \\
\hline
\end{tabular}

Tableau III. Principales molécules produites chez les microalgues.

reinhardtii mais aussi chez la diatomée Cylindrotheca fusiformis. Dans la voie nucléaire, l'événement le plus courant est une recombinaison dite non homologue. Plusieurs techniques ont tenté de favoriser l'intégration en agrandissant la taille des vecteurs ou en incluant des marqueurs de sélection particuliers, mais dans la plupart des cas l'on observe que quelques intégrations aléatoires dans le génome (intégrations ectopiques). Des travaux déterminants sur ce sujet ont été publiés et une piste pourrait consister à identifier un site silencieux à l'exemple de Chlamydomonas reinhardtii. La transformation chloroplastique a été décrite pour la première fois chez l'algue unicellulaire Chlamydomonas reinhardtii. Les promoteurs provenant des gènes chloroplastiques tels $r b c L$ (ribulose bisphosphate carboxylase/oxygènase sous-unité majeure) et atpA (subunité $\lambda$-ATP synthase) de Chlamydomonas reinhardtii sont les plus fréquents. Les gènes rapporteurs couramment utilisés pour les études d'expression des gènes dans le chloroplaste sont le gène gfp (green fluorescent protein, GFP), luxCt (Luciferase, LUC) et le gène uidA (GUS). Ces marqueurs fonctionnent dans d'autres groupes d'algues, comme par exemple la diatomée Phaeodactylum tricornutum. Une telle entreprise bénéficie grandement des avancées qu'offre la publication des données de séquençages complets des génomes. La liste s'étoffe et nous avons dorénavant accès aux génomes complets de Chlamydomonas reinardtii, Cyanidoschison merolae, Thalassiosira pseudonana, Ostreococcus taurii et Phaeodactylum tricornutum en cours d'annotation et à laquelle nos équipes participent.

\section{Conclusion}

L'industrie pharmaceutique et les sociétés de biotechnologie sont confrontées à un défi majeur : celui de disposer de systèmes de production de protéines recombinantes qui puissent répondre à leurs contraintes à la fois en terme de qualité, de quantité produite et de rentabilité. Il existe au niveau mondial un déficit de capacité de production de ces protéines recombinantes, car les systèmes actuellement disponibles ne permettent pas de produire en quantité suffisante des protéines de qualité pour un coût raisonnable. Ce besoin va s'accentuer dans les années à venir. Depuis la fin des années 1990, plus de 280 protéines recombinantes thérapeutiques, dont une vingtaine d'anticorps monoclonaux, sont commercialisées, représentant un chiffre d'affaires estimé à près de 49 milliards de dollars US en 2006. Ce domaine d'activité connaît une croissance de $20 \%$ par an contre $7 \%$ pour l'industrie pharmaceutique globale et près de 2200 nouvelles protéines recombinantes sont actuellement en phase clinique. L'idée de créer une structure de production de molécules recombinantes à haute valeur commerciale dans un système microalgue a déjà conduit à la création de deux sociétés aux États-Unis. La stratégie est tangible et son succès est exclusivement dépendant de choix judicieux en termes d'espèces de microalgues, de molécules exprimées et de ressource humaine. $\diamond$

\section{SUMMARY}

Microalgae as cell factories

producing recombinant commercial proteins

Extraction of natural substances and chemical synthesis are the main sources of pharmaceutical molecules. When possible, one may transfer the gene of the molecule in living cells creating individual factories producing on demand and in a safe way the requested molecule. Today, bacteria, yeast, mammalian cells and plants constitute the main plate-forms for various commercial products. Microalgae present numerous advantages and could offer a powerful tool for the production of commercial molecules in a near future. $\diamond$

Légende des figures 1 à 4 (photos): Phaeodactylum tricornutum exprimant la Green Fluorescent Protein (c) A. Carlier/Ifremer).

\section{REMERCIEMENTS}

Nous tenons à remercier les deux évaluateurs anonymes pour leur aide et critiques constructives

\section{RÉFÉRENCES}

1. Schmidt FR. Recombinant expression systems in the pharmaceutical industry. Appl Microbiol Biotechnol $2004 ; 65$ : 363-72.

2. Hunt I. From gene to protein: a review of new and enabling technologies for multi-parallel protein expression. Protein Expr Purif $2005 ; 40$ : 1-22.

3. Sodoyer R. Expression systems for the production of recombinant pharmaceuticals. Biodrugs $2004 ; 18: 51-62$.

4. Lee $\varepsilon$, Roth J, Paulson JC. Alteration of terminal glycosylation sequences on $\mathrm{N}$-linked oligosaccharides of Chinese hamster ovary cells by expression of beta-galactoside alpha 2,6-sialyltransferase. J Biol Chem $1989 ; 264: 13848-55$.

5. Paccalet T, Bardor M, Rihouey C, et al. Synthesis of sialic acid in plants. Plant Biotech J 2007 ; 5: 12-25.

6. Hamilton SR, Davidson RC, Sethuraman N, et al. Humanization of yeast to produce complex terminally sialylated glycoproteins. Science 2006 ; $313: 1441-3$

7. Hiatt A, Caffferkey R, Bowdish C. Production of antibodies in transgenic plants. Nature $1989 ; 342: 76-8$.

8. Ma JKC, Chikwarnba R, Sparrow P, et al. Plant-derived pharmaceuticals - the road forward. Trends Plant Sci $2005 ; 10: 580-5$.

9. Bardor M, Faveeuw C, Fitchette AC, et al. Immunoreactivity in mammals of two typical plant glyco-epitopes, core alpha $(1,3)$-fucose and core xylose. Glycobiology $2003 ; 13$ : 427-34. 
10. Saint-Jore-Dupas C, Faye L, Gomord V. From planta to pharma with glycosylation in the toolbox. Trends Biotechnol $2007 ; 25: 317-23$

11. Weise A, Altman F, Rodriguez-Franco M, et al. High-level expression of secreted complex glycosylated recombinant human erythropoietin in the Physcomitrella. Plant Biotechnol J $2007 ; 5: 389-401$.

12. Frank W, Decker EL, Reski R. Molecular tools to study Physcomitrella patens. Plant Biol 2005 $7: 220-7$.

13. Stevens DR, Purton S. Genetic engineering of eukaryotic algae: Progress and prospects. J Phycol 1997 ; $33: 713-22$

14. Leon-Banares R, Gonzalez-Ballester D, Galvan A, Fernandez $\varepsilon$. Transgenic microalgae as green cell-factories. Trends Biotechnol $2004 ; 22: 45-52$.

15. Franklin SE, Mayfield SP. Recent developments in the production of human therapeutic proteins in eukaryotic algae. Ext Opin Biol Ther $2005 ; 5: 225-35$.

16. Walker TL, Collet C, Purton S. Algal transgenics in the genomic ERA. J Phycol 2005; 41 : 1077-93.

17. Walker TL, Purton S, Becker DK, Collet C. Microalgae as bioreactors. Plant Cell Rep 2005 ; $24: 629-41$.

18. Harris EH. Chlamydomonas as a model organism. Annu Rev Plant Phys 2001 ; $52: 363-406$.

19. Kim DH, Kim YT, Cho JJ, et al. Stable integration and functional expression of flounder growth hormone gene in transformed microalga, Chlorella ellipsoidea. Marine Biotechnol 2002 ; $4: 63-73$.

20. Debuchy R, Purton S, Rochaix JD, The arginosuccinate lyase gene of Chlamydomonas reinhardtii: an important tool for nuclear transformation and for correlating the genetic and molecular maps of the ARG7 locus. EMBO J $1989 ; 8$ : 2803-9.

21. Boynton JE, Gillham NW, Harris $\varepsilon H$, et al. Chloroplast transformation in Chlamydomonas with high velocity microprojectiles. Science $1988 ; 240: 1534-8$.

22. Randolph-Anderson BL, Boynton JE, Gillham NW, et al. Further characterization of the respiratory deficient dum-1 mutation of Chlamydomonas reinhardtii and its use as a recipient for mitochondrial transformation. Mol Gen Genet 1993; 236 : 235-44.

23. Brown LE, Sprecher SL, Keller LR. Introduction of exogenous DNA into Chlamydomonas reinhardtii by electroporation. Mol Cell Biol 1991; 11 : 2328-32.

24. Kindle K. High-frequency nuclear transformation of Chlamydomonas reinhardtii. Proc Natl Aca Sci USA $1990 ; 87: 1228-32$.

25. Dunahay TG. Nuclear transformation of Chlamydomonas reinhardtii with silicon carbide fibers. J Phycol $1992 ; 28: 11$

26. Kumar SV, Misquitta RW, Reddy VS, et al. Genetic transformation of the green alga Chlamydomonas reinhardtii by Agrobacterium tumefaciens. Plant Science $2004 ; 166: 731-8$.

27. Geng DG, Wang YQ, Wang $P$, et al. Stable expression of hepatitis $B$ surface antigen gene in Dunaliella salina (Chlorophyta). J Appl Phycol 2003 ; 15 : 451-6.

28. Tan C, Qin S, Zhang $Q$, et al. Establishment of a micro-particle bombardment transformation system for Dunaliella salina. J Microbiol $2005 ; 43: 361-5$.

29. Jarvis $દ \varepsilon$, Brown LM. Transient expression of firefly luciferase in protoplasts of the green alga Chlorella ellipsoidea. Curr Genet 1991; 19 : 317-21.

30. Dawson HN, Burlingame R, Cannons AC. Stable transformation of Chlorella: rescue of nitrate reductase-deficient mutants with the nitrate reductase gene. Curr Microbiol 1997 ; $35: 356-62$.

31. Chow K, Tung WL. Electrotransformation of Chlorella vulgaris. Plant Cell Reports 1999 ; $18: 778-80$.
32. Teng CY, @in S, Liu JG, et al. Transient expression of lacZ in bombarded unicellular green alga Haematococcus pluvialis. J Appl Phycol 2002 ; $14: 497-500$.

33. Scheidlmeier B, Schmitt R, Müller W, et al. Nuclear transformation of Volvox carteri. Proc Natl Acad Sci USA 1994 ; 91 : 5080-4.

34. Dunahay TG, Jarvis $\varepsilon \varepsilon$, Roessler PG. Genetic transformation of the diatoms Cyclotella cryptica and Navicula saprophila.J Phycol 1995 31: 1004-12

35. Apt KE, Kroth-Pancic PG, Grossman AR. Stable nuclear transformation of the diatom Phaeodactylum tricornutum. Mol Gen Genet 1996 ; $252: 572-9$.

36. Fischer H, Robl I, Sumper M, Kroger N. Targeting and covalent modification of cell wall and membrane proteins heterologously expressed in the diatom Cylindrotheca fusiformis (Bacillariophyceae). J Phycol 1999; 35: 113-20.

37. Poulsen N, Kroger N. A new molecular tool for transgenic diatoms: control of mRNA and protein biosynthesis by an inducible promoter-terminator cassette. Febs Lett $2005 ; 272$ : 3413-23.

38. ten Lohuis MR, Miller DJ. Light-regulated transcription of genes encoding peridinin chlorophyll a proteins and the major intrinsic lightharvesting complex proteins in the dinoflagellate Amphidinium carterae hulburt (Dinophycae). Changes In cytosine methylation accompany photoadaptation. Plant Physiol 1998; 117 : 189-96.

39. Minoda A, Sakagami R, Yagisawa F, et al. Improvement of culture conditions and evidence for nuclear transformation by homologous recombination in a red alga, Cyanidioschyzon merolae. Plant Cell Physiol $2004 ; 45: 667-71$.

40. Lapidot M, Raveh D, Sivan A, et al. Stable chloroplaste transformation of the unicellular red alga Porphyridium species. Plant Physiol 2002 ; $129: 7-12$.

41. Doetsch NA, Favreau MR, Kuscuoglu N, et al. Chloroplast transformation in Euglena gracilis: splicing of a group III twintron transcribed from a transgenic psbK operon. Curr Genet $2001 ; 39: 49-60$.

42. Mayfield SP, Franklin SE, Lerner RA. Expression and assembly of a fully active antibody in algae. Proc Natl Acad Sci USA $2003 ; 100: 438-42$.

43. Manuell AL, Beligni MV, Elder JH, et al. Robust expression of a bioactive mammalian protein in Chlamydomonas chloroplast. Plant Biotechnol J $2007 ; 5: 402-12$.

44. Sun M, Pian K, Su N, et al. Foot-and-mouth disease virus VPl protein fused with cholera toxin B subunit expressed in Chlamydomonas reinhardtii chloroplast. Biotechnol Lett $2003 ; 25$ : 1087-92.

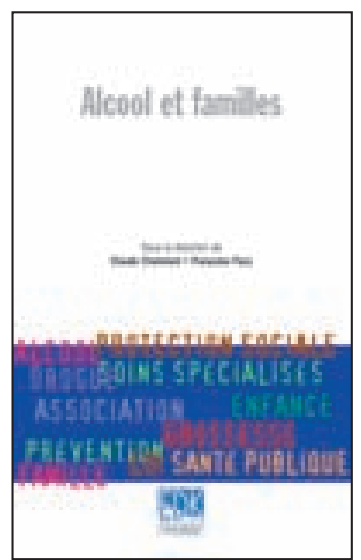

ISBN : 978-2-8425-4116-3 174 pages

\section{Bon de commande}

À retourner à EDK, 2, rue Troyon - 92316 Sèvres Cedex

Tél. : 0155641393 - Fax : 0155641394 - E-mail : edk@edk.fr

NOM : Prénom :

Adresse :

Code postal : Ville :

Pays :

Fonction :

Je souhaite recevoir l'ouvrage Alcool et familles : $15 €+3 €$ de port $=\mathbf{1 8} €$ TTC

en .. exemplaire, soit un total de $€$

Par chèque, à l'ordre de $\mathbf{E} \mathbf{D} \mathbf{K}$

Par carte bancaire : $\quad$ Visa $\quad$ Eurocard/Mastercard

Carte $\mathrm{n}^{\circ}$

Date d'expiration :

$\mathrm{N}^{\circ}$ de contrôle au dos de la carte :

\section{TIRÉS À PART}

J.P. Cadoret 